\title{
Kangaroo Mother Care Implementation at the Philippine General Hospital: A Quality Assurance Initiative
}

\author{
Maria Esterlita T. Villanueva-Uy, MD, MSPH, ${ }^{1,2,3}$ Lucille Marie Villanueva-Uy, ${ }^{4,5}$ \\ Andrea Lauren Tang Chung ${ }^{4,6}$ and Socorro De Leon-Mendoza, MD $^{3}$ \\ ${ }^{1}$ Institute of Child Health and Human Development, National Institutes of Health, University of the Philippines Manila \\ ${ }^{2}$ Section of Nerwborn Medicine, Department of Pediatrics, Philippine General Hospital, University of the Philippines Manila \\ ${ }^{3}$ Kangaroo Mother Care Foundation Philippines, Inc. \\ ${ }^{4}$ Immaculate Conception Academy, Greenhills, San Juan City \\ ${ }^{5}$ College of Medicine, University of the Philippines Manila \\ ${ }^{6}$ Ateneo de Manila University, Quezon City
}

\section{ABSTRACT}

Background. The Philippine General Hospital (PGH) implemented the Kangaroo Mother Care (KMC) Program in 2014, recognizing its benefits in helping low birth weight (LBW) infants survive.

Objective. To determine the acceptability and compliance of the stakeholders to the KMC program after one year from implementation.

Method. Data were obtained from the NICU Annual statistics, KMC data forms, logbooks, and questionnaires to the stakeholders-doctors rotating at the NICU, NICU nurses, and mothers enrolled in the KMC program.

Results. One year into the KMC program implementation, the KMC enrollment increased from $57 \%$ in 2014 to $75 \%$ in 2015. All mothers enrolled in the program said that they received their KMC knowledge from the health providers and firmly believed that KMC benefited them and their infants. The mothers also became more confident in taking care of their babies after each KMC encounter. Although only 50\% said they would continue KMC at home, $85 \%$ proceeded.

Furthermore, both doctors and nurses believed that KMC was beneficial to both mothers and infants, decreased hospital cost and nursing workload. KMC provision was 0.5-6 hours/day. Also, less than half of the data forms were accomplished.

The KMC program was acceptable to all stakeholders who believed in the benefits of KMC to preterm infants. The mothers were very receptive and continued KMC even after discharge. However, there was sub-optimal engagement provided by the health providers with the mothers. There was also low adherence to recommended duration of KMC per day provided by the mothers. KMC data records were frequently not accomplished. PGH has instituted strategies

Poster presented in the $11^{\text {th }}$ International Workshop and Congress of Kangaroo Mother Care on November 14-17, 2016, at Trieste, Italy.

This article expounded on the findings of an interim quality improvement assessment of the KMC program, which was undertaken to determine the perspectives of all stakeholders - the medical and nursing KMC providers and the mothers who provided KMC to their infants - with regards their KMC experience. In addition, enablers, and bottlenecks specific to the PGH settings but relevant to other local and national KMC concerns, were also enumerated.

Corresponding author:

Maria Esterlita T. Villanueva-Uy, MD, MSPH

Section of Newborn Medicine, Department of Pediatrics

Philippine General Hospital, University of the Philippines Manila

Taft Avenue, Ermita, Manila 1000, Philippines

Email: mvuy1@up.edu.ph to improve the $\mathrm{KMC}$ implementation by providing dedicated $\mathrm{KMC}$ rooms and supplying meals to mothers to increase KMC duration and frequency. A computerbased program for data entry was developed for the health providers, and a dedicated encoder was assigned.

Conclusion. KMC acceptability was high among stakeholders. Compliance increased after one year, with enrolment going up to $75 \%$. However, adherence to the recommended KMC duration per day and accomplishment of data forms were still sub-optimal.

Key Words: Kangaroo mother care program, implementation, Philippine General Hospital 


\section{INTRODUCTION}

Kangaroo Mother Care (KMC) is a recognized intervention in helping low birth weight (LBW) infants (preterm and small infants) survive. ${ }^{1}$ Despite robust evidence, the implementation of $\mathrm{KMC}$ has faced several barriers in several multi-country assessment studies, particularly in Asian countries. ${ }^{2}$ Based on the consensus reached after the 2013 International KMC conference in Istanbul, Turkey, reasons cited for the lag in the implementations were: ${ }^{2}$

- $\mathrm{KMC}$ incorrectly perceived as a practice for preterm newborns in low-income countries only, as a "next best" alternative to incubator care.

- Many healthcare providers (at all levels) do not know or do not believe in the benefits of KMC and lack the skills for effective implementation.

- Cultural and social norms related to mother and newborn practices make the uptake of $\mathrm{KMC}$ challenging.

- Human resources for health required for KMC have been lacking, and the role of mothers and communities has been overlooked.

- KMC has not been included in many country-level government newborn agendas and policies.

The Philippine General Hospital (PGH), the national university hospital, is a tertiary referral center catering to the country's different regions. The average number of annual live births from 2014-2016 was 6,092 live births. ${ }^{3-5}$ The Neonatal Intensive Care Unit (NICU) has a 40-bed capacity but is often exceeded, at times, accommodating as many as 100 sick infants. Since the PGH NICU cares for high-risk infants, the preterm rate had steadily increased from $10 \%$ in 2004 to $19.6 \%$ in 2016 . LBW ( $<2,500$ grams) is high at $33 \%$ of live births in 2016 compared with the national LBW rate of $21 \%{ }^{6}$

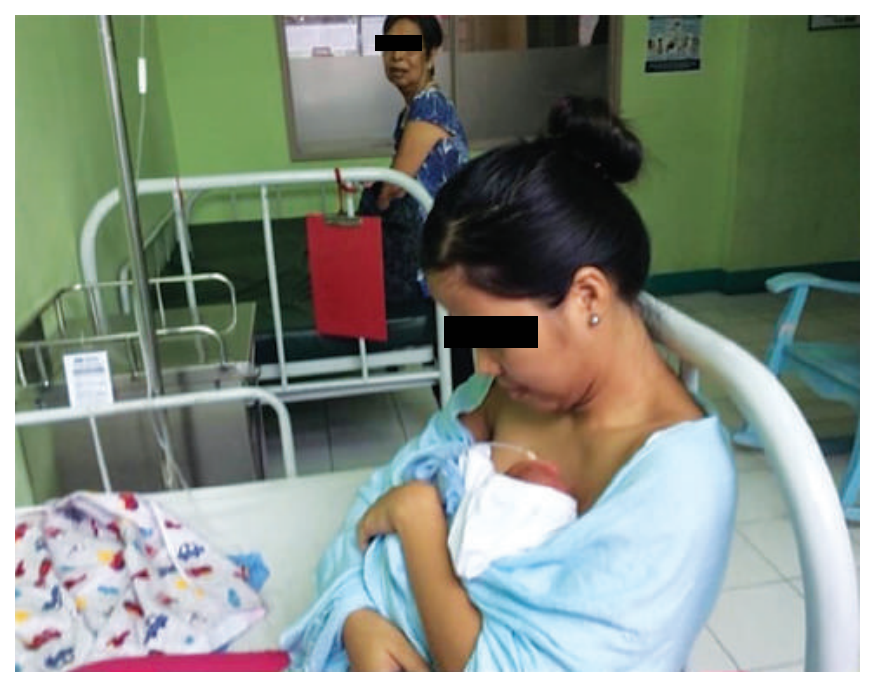

Figure 1. Mother and infant on KMC position in the Kiko's room.
KMC was informally introduced to the PGH NICU by conducting several Neonatology Fellow-led clinical trials on KMC from 2010 until the present. Recognizing the benefits of KMC based on local research evidence, the NICU doctors and nurses had slowly integrated its practice in daily neonatal care. Research collaborations with the KMC Foundation Philippines provided the opportunity for further familiarization with the KMC intervention. Lastly, the call for accelerated action for KMC implementation published in the scientific journal - Lancet in its NovemberDecember 2013 issue, ${ }^{7}$ set the impetus to undergo a Trainingof-Trainer's workshop conducted by the KMC Foundation Philippines. This steered to the formal implementation of the Kangaroo Mother Care program at PGH in 2014. A KMC committee was created to guide the application of the KMC program in the different units caring for the LBW infants - Neonatal Intensive Care Unit- (Levels 2 and 3), Pediatric Intensive Care Unit, Kiko's Room (4-bed KMC ward) (Figure 1), Pediatric and Rooming-in wards.

The initial KMC intervention in the PGH NICU was through intermittent KMC (at least 6 hours of KMC per day) due to the absence of dedicated rooms for the parents to stay for 24 hours. Rocking chairs and lounge chairs were available in one corner of the NICU to provide privacy for the mothers rendering $\mathrm{KMC}$ (Figure 2). In the

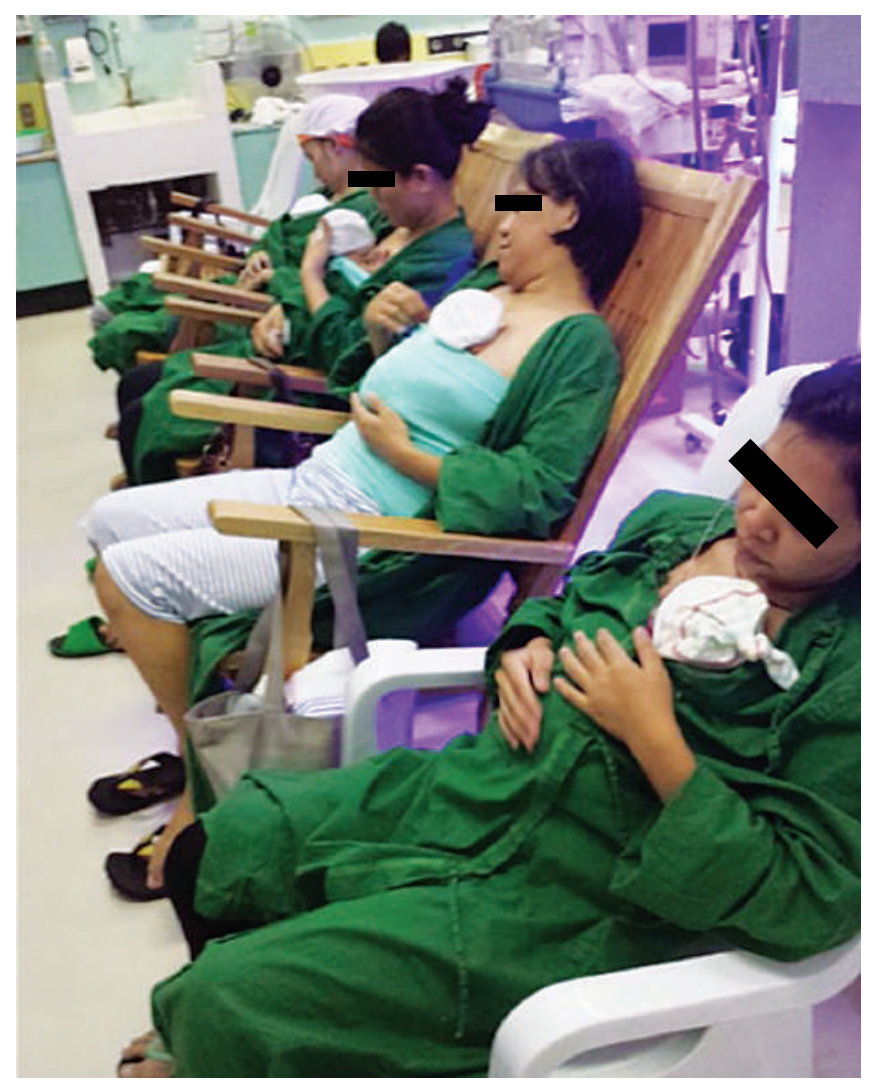

Figure 2. Mothers providing intermitted $\mathrm{KMC}$ to their infants at the PGH NICU. 
PICU, intermittent KMC was rendered at the bedside. Continuous KMC ( $\geq 18$ hours a day) was encouraged for direct rooming-in and private rooms, but implementation was not strictly monitored. Upon discharge, the mothers were instructed to continue $\mathrm{KMC}$ in their homes.

\section{Social Value}

There is robust evidence that $\mathrm{KMC}$ has led to a substantial reduction in preterm mortality. Still, there was a lag in its implementation. An accelerated action for KMC programs was called worldwide with a goal of at least 50\% of LBW infants provided KMC by $2020 .^{7}$ In Southeast Asia, cascading of the KMC training to different hospitals have started. However, implementation was still described as patchy or variable. ${ }^{8}$ It is thus vital to have an interim assessment of the KMC program at the $\mathrm{PGH}$ to implement it optimally.

\section{OBJECTIVE}

After one year of implementation, we would like to obtain an interim assessment of the KMC program about acceptability to the stakeholders and compliance.

\section{METHODS}

As part of a quality improvement assessment of the program, questionnaires were given to several stakeholders. In one month (May 2015), the Pediatric residents were rotating at the NICU; NICU nurses were approached and asked to answer a self-administered KMC perception and compliance questionnaire. Mothers who were providing $\mathrm{KMC}$ in the NICU were also approached and invited to answer a similar questionnaire. Furthermore, in the HighRisk Clinic, mothers who followed up the High-Risk Clinic and were enrolled in the KMC program during their infants' hospitalization were also approached and asked to answer a similar questionnaire. Pediatric residents in the High-Risk Clinic were also requested to answer a similar survey.

There are data forms used by local and foreign hospitals which have KMC programs. These forms were obtained from the Kangaroo Mother Care International Network. After proper orientation, the documents were made available to health care providers for data entry. The KMC data forms consisted of the in-patient and outpatient data forms, which were both accomplished by the doctors. Another form assessing maternal adaptation to the KMC practice was completed by the nurses in charge of the infants. These KMC data forms were reviewed for the completion of entries.

Other data sources reviewed were the PGH Annual NICU statistics to determine the enrollment rate to the KMC program..$^{3-5,9}$ The mother's logbook, which records exit and entry to the NICU, was also reviewed to determine the length of $\mathrm{KMC}$ rendered.

\section{RESULTS}

Based on the available annual NICU statistics, KMC enrolment was $57 \%$ of all eligible preterm and low birth weight infants before the program's implementation in 2014. One year after implementation, the enrollment rate increased to $75 \%$ in 2015 .

\section{KMC mothers}

Mothers who were enrolled in the KMC program claimed that they were introduced to the concept of KMC primarily by the nurses $(57.14 \%)$, followed by the doctors (23.81\%) or both (16.7\%). However, almost half (47.62\%) divulged that they did not receive additional information about $\mathrm{KMC}$ anymore from any health provider during their subsequent KMC encounters. There seemed to be less interaction between the health staff and the mothers of the enrolled babies regarding day-to-day KMC encounters. Despite this, over $80 \%$ of the mothers felt that the nurses were beneficial during their $\mathrm{KMC}$ encounters.

Mothers rendering $\mathrm{KMC}$ in the hospital were knowledgeable about the primary purpose of the KMC encounter, which was to keep the baby warm. However, the mothers were not familiar with the other benefits of KMC on maternal-infant bonding, growth, and decrease in infection. Still, all felt happy providing KMC to their infants and accepted integrating the practice into their daily lives. All believed that their husbands would be happy also to render $\mathrm{KMC}$ to their children. Due to the distance of their homes to PGH and the lack of funds, almost half of the mothers disclosed that they had a hard time getting to PGH, and thus they were unable to make daily visits to their children. Only $50 \%$ of the mothers intended to continue KMC after the discharge of their infants.

On follow-up at the High-Risk Clinic, a majority (85.71\%) of mothers turned out to continue KMC up to the time of follow-up visit while $7.14 \%$ stopped $\mathrm{KMC}$ once the infant was discharged and another 7.14\% stopped KMC once the infant reached $2.5 \mathrm{~kg}$. Again, according to all mothers at the High-Risk Clinic, they believed that KMC played a role in their infants' faster growth and earlier discharge. However, only $57 \%$ of the mothers interviewed recalled information on KMC during their infant's confinement. More so, the mothers claimed that this was done infrequently.

\section{Health Providers}

All doctors and nurses in the NICU were aware of the KMC program. Seventy percent learned first about KMC during their NICU rotation, while the others knew about the program from colleagues (19\%) and outside lectures (11\%). Similarly, the fora where they learned about KMC were during a PGH training workshop (33\%), discussion with colleagues (28\%), lectures (23\%), nursing workshop (9\%), radio/TV (5\%), and NICU audits (2\%). 
The majority of doctors and nurses agree that KMC does indeed help babies grow faster and healthier, lessens hospital costs, and decreases the workload. The KMC program included activities such as calling the mothers to come to the NICU, teaching $\mathrm{KMC}$ to the mothers, placing the infant on the mother's chest, and filling up the KMC data records. The nurses liked placing the infant on the mother's chest (KMC position) the most, while the doctors preferred teaching the mothers about $\mathrm{KMC}$. Both doctors and nurses least liked accomplishing $\mathrm{KMC}$ records.

\section{Completion of KMC forms}

Twenty-seven KMC data forms of discharged infants were available for review during the assessment period. Only $45.6 \%$ of the data forms for in-patient data had entries, although incomplete, while the rest were entirely blank. The portion of the in-patient data forms with entries were the general data, prenatal and intrapartum data, which the Pediatric residents usually accomplished during the infant's admission to the NICU. Data of the clinical course mainly were left unfilled. This indicated that the Pediatric residents no longer return to the forms after the first entry. The possible reasons for non-compliance were non-compulsory completion of sheets, change of residents rotating in the NICU during the infant's stay, transfer of the infant from the NICU to the step-down unit, which has a different set of residents, and irregular monitoring of the data forms.

A review of the outpatient KMC forms again showed that only $46 \%$ of the forms were accomplished, and these mainly consisted of the patient's general information. There was hardly any information written on the more medically focused area.

The maternal adaptation form contained the nurses' observations on how the mother rendered KMC, duration of $\mathrm{KMC}$, infant weight gain, breastmilk production, response to feeding cues, family support, and baby's reactions. The mothers were observed, interviewed, or both every time they rendered KMC. Almost $65 \%$ of the forms were completed. However, the trend was that after three days of entries, the nurses stopped entering data. Still, this was the KMC form with the highest entry compliance.

\section{DISCUSSION}

As a whole, the PGH KMC program has been most welcomed by the mothers who now have the opportunity to hold their infants in the KMC position for a longer duration daily. They learned about the significant benefits of $\mathrm{KMC}$ and were happy to provide $\mathrm{KMC}$ to their children. The majority continued rendering KMC to their infants even after hospital discharge. They were enthusiastic about the program and even desired more information about KMC from the health providers when they render KMC to the infants in the hospital. Unlike in the study of Bilal on $\mathrm{KMC}$ implementation in Ethiopia, there were problems with acceptance of the KMC by mothers due to cultural, traditional, and family beliefs that a preterm infant is a stigma, will not survive, and lack of awareness to the KMC. ${ }^{10}$

The maternal compliance is high, but the $\mathrm{KMC}$ duration was below the recommended hours. To improve the duration of KMC provided daily, PGH provides the mothers with meals whenever they go to the hospital for KMC. After discharge, the preterm infants are followed up at the High-Risk Clinic to monitor KMC compliance and identify problems associated with prematurity. HighRisk follow-up continues up to 1 year old, and infants are subsequently transferred to the Well Baby Clinics. The mothers were also given a crib donated by the World Health Organization once their children reached one year old as a symbol of 'KMC graduation.' Consequently, PGH started holding Annual Preemie Reunions to engage with the mothers and former preterm infants again.

The doctors and the nurses are an integral part of the KMC program. They are knowledgeable about the benefits of $\mathrm{KMC}$. However, daily interaction with the mothers renders $\mathrm{KMC}$ is sub-optimal. The daily $\mathrm{KMC}$ teaching and reassurance to the mothers need to be optimized as the mothers felt that this was the weakest part of the program. Similar to the Ethiopian study, doctors and nurses need to improve in their engagement with the mothers. ${ }^{9}$ Since the mothers are receptive to the $\mathrm{KMC}$ program, the doctors and nurses should be motivated to ensure that the babies receive KMC optimally - long duration, daily encounters, and beyond discharge. Regular training workshops on KMC may help encourage doctors and nurses to increase support to the mothers providing KMC. Development of guidelines and detailed instructions on enrollment and continuance of the mother-infant dyads in the KMC program may encourage better communication and interaction with the doctors and nurses. One of the essential vital factors for implementation success was the training of all health care staff on the KMC protocol, including eligibility criteria, duration of KMC until follow-up in the clinics. ${ }^{11}$

KMC data forms are part of the country's assessment program. These forms, which originated from the Fundacion Canguro of Colombia, are used by several countries to assess KMC program implementations. Unfortunately, an accomplishment of the KMC data forms at PGH greatly needs improvement. Less than half of the forms had entries, albeit incomplete. The KMC inpatient forms are readily available in the NICU. Still, data input is required at several time points during the infant's hospital stay - admission, resolution of illness, new problems, feeding advancements, medications, and discharge. As such, most forms will have the admission data, but the Pediatric residents may not return and update the forms regarding other events - new and resolved illnesses, medication duration, type of feedings, etc. There will be a new Pediatric resident in charge at times, and the forms may not be continued. Strategies to improve compliance were developing a computer program for encoding 
data and assigning a dedicated person to input the data. This has led to the increase in the data forms accomplished temporarily, but when the task was transferred back to the residents, forms were again incompletely completed. One possible strategy is the integration of the KMC data forms into the hospital's electronic medical records.

\section{Bottlenecks in the Implementation of KMC and the PGH response}

In a 12-country analysis of bottlenecks in the implementation of KMC published in 2016, 3 major areas were identified, which were especially true in Southeast Asia. ${ }^{2}$ Some of these are relevant to the PGH setting and the Philippines as a whole. The first barrier identified was health financing, in which national budgets for neonatal care were deemed suboptimal. Hospital costs and loss of income are high when caring for a preterm infant. Recognizing this, the government-owned insurance corporation, PhilHealth, developed a prematurity package that included KMC as a requisite intervention in preterm care. The prematurity package was created to provide a financial scheme to reimburse the participating hospitals and set limits to PhilHealth members' hospital payments. PGH applied as a Health Care Institution (HCI) for the prematurity package, which would significantly help the indigent patients the hospital caters to.

A significant bottleneck identified in the multi-country assessment was the inadequate health delivery systems. Quality KMC means KMC is provided continuously for at least 18 hours, initiated early, and continued even after hospital discharge. Based on the KMC mother's logbook at the PGH NICU, the duration of KMC provided by the mothers was inconsistent, ranging from a few minutes to 6 hours a day. Meals are now given to the mothers, and a dedicated KMC room has been provided where the mothers can stay 24 hours a day. As shown in the 1-year PGH assessment, there should be continued interaction between the health provider and mother enrolled in the KMC program to encourage quality KMC. Another weakness of the PGH KMC program was the inadequate data recording. Currently, a dedicated encoder is entering the $\mathrm{KMC}$ data needed to assess the different KMC programs in the country.

Another bottleneck identified was the lack of community ownership, described as a lack of community awareness and mobilization regarding $\mathrm{KMC}$. As seen in the 1-year PGH assessment, KMC awareness of the mothers only came from the hospital staff and none from other social media. Similarly, the information of the doctors was obtained within the hospitals'workshops. The topic on KMC is currently included in the Lactation Management Training course, the KMC training workshop, the Care for the Small Baby training course, and even the Neonatal Resuscitation Program held at PGH other hospitals. Loop videos on KMC are also played regularly in the key areas of the hospital. However, the information of KMC has not reached relevant social media vehicles to educate the community.
In a systematic review of Chan and Smith, the lack of time to perform $\mathrm{KMC}$ was obstructing $\mathrm{KMC}$ implementation. ${ }^{12,13}$ In our study, the data forms were not accomplished, possibly due to a lack of time to encode the data. Since the PCH NICU's occupancy rate is almost always beyond the allotted bed capacity, the nursing and resident doctor staff may prioritize management of sick infants rather than provide time to enter the $\mathrm{KMC}$ data. Implementing strategies may include dedicated time for writing progress notes and $\mathrm{KMC}$ forms, trimming down the data needed in the $\mathrm{KMC}$ forms, and, as was done at $\mathrm{PGH}$, a reliable person to encode the data. The last option would entail additional job orders from the hospital.

\section{Scaling up KMC at PGH}

Three strategies have been identified to scale up KMC in the hospital and community in the multi-country $\mathrm{KMC}$ analysis. ${ }^{2}$ One is the presence of $\mathrm{KMC}$ champion/s to initiate or improve implementation of the program. At the $\mathrm{PGH}$, other strategies to strengthen the $\mathrm{KMC}$ program were undertaken. With the leadership of the PGH Medical Director, Dr. Gerardo Legazpi, a designated KMC room adjacent to the NICU was allocated to allow for continuous KMC provision of the mothers to their stable preterm infants. The private hospital room was renovated and subsequently inaugurated with the help of the Metro Manila Mayors' Spouses Foundation (Figure 3). The KMC room (Room 400) can accommodate five mothers who can stay with their stable preterm infants 24/7. They are provided with an adjustable bed that can facilitate $\mathrm{KMC}$, designed by the PGH Medical Director (Figure 4). The room has an air conditioner and has a bathroom. This strengthens the mother-infant bonding and, at the same time, decongests the main NICU, which is often overcrowded. Moreover, with the permission of then Pediatric Department Chair, Dr. Juliet Sio Aguilar, another room (Kiko's room) located at the end of the Pediatric ward has been converted into a convalescent or step-down area for out born stable preterm infants who were initially admitted in the wards for acute illnesses.

Second, the scaling up of the KMC program maybe through a project-initiated approach. KMC training workshops were conducted through grants from Save the Children and the Department of Health (DOH). Several Neonatology fellows led clinical trials to increase awareness and implementation of KMC in the NICU. Collaboration with the KMC Foundation Philippines, a non-government organization, has allowed the conduct of the study and presentation of results locally and internationally.

Third, health system-designed programs from the national government lack in other countries. Fortunately, the DOH spearheaded the development of the National Policy on the Quality Care of the Small Baby, of which $\mathrm{PGH}$ has been compliant. After passing the eligibility assessment, PGH was awarded as a KMC Center of Training and Excellence (Figure 5). Together with the KMC 


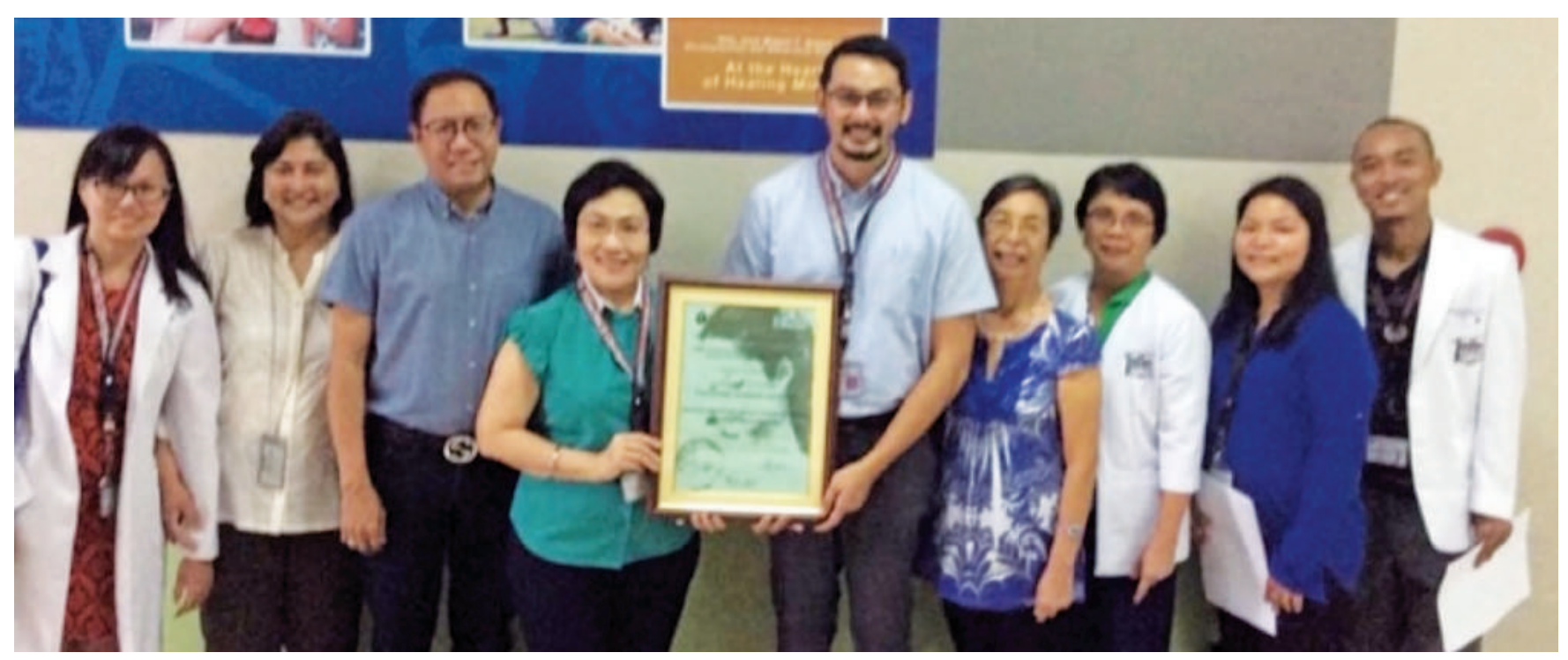

Figure 3. PGH awarded as KMC Center of Excellence. From left to right: Dr. Shary Baton (Neonatology Fellow), Dr. Maria Esterlita V. Uy (Head, KMC Committee), Dr. Wilfredo Santos (PPS Board of Trustees), Dr. Juliet Sio Aguilar (then Chair of UP Department of Pediatrics UP-PGH), Dr. Resti Ma. Bautista, (Head of the Division of Newborn Medicine, UP-PGH), Annabella Guerrero (Board Member, KMC Foundation Philippines), Fe Basinang RN (Head Nurse, PGH-NICU), Dr. Fay de Ocampo (Neonatologist, Division of Newborn Medicine, UP-PGH) and Dr. Ardie Lugo (Neonatology Fellow).

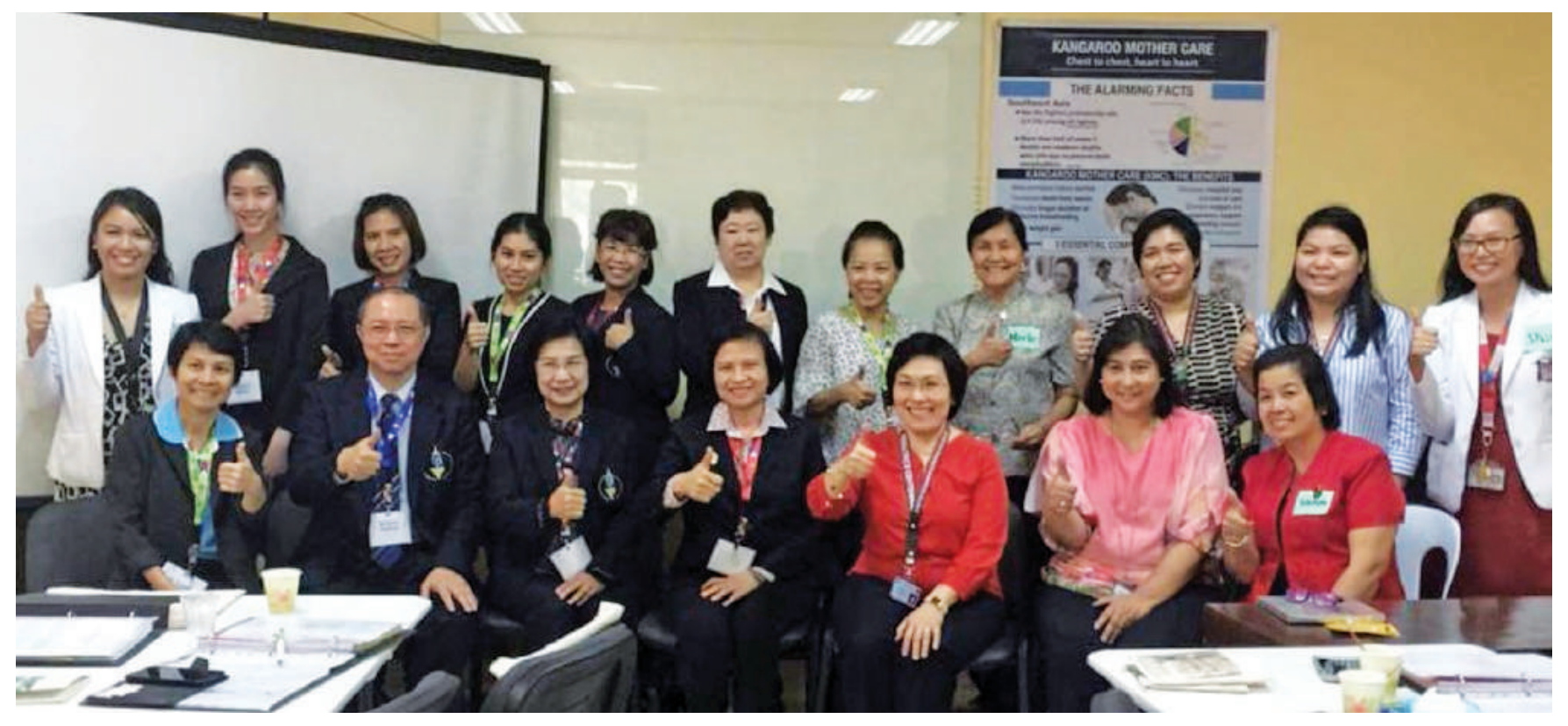

Figure 4. KMC Trainers' Workshop conducted by PGH to the medical and nursing staff of Queen Sirikit Institute of Child Health, Bangkok, Thailand. The workshop was held at the Ortoll Building, Philippine General Hospital.

Foundation Philippines, PGH has conducted a KMC trainers' workshop requested by the doctors and health staff of the Queen Sirikit National Institute of Child Health, Bangkok, Thailand, in 2016 (Figure 6).

Another enabler at the $\mathrm{PGH}$ is the introduction of $\mathrm{KMC}$ awareness to the undergraduate and medical students in the form of a one-month elective rotation, mentoring activity, or a summer internship. These activities have been met with positive feedback and have produced at least two research papers. In the mentoring training, the medical students were asked to design the plain KMC tubes. After which, with the mothers' permission, the students wearing the $\mathrm{KMC}$ tubes they created, provided $\mathrm{KMC}$ to the stable preterm infants (Figure 7). Although apprehensive initially, 


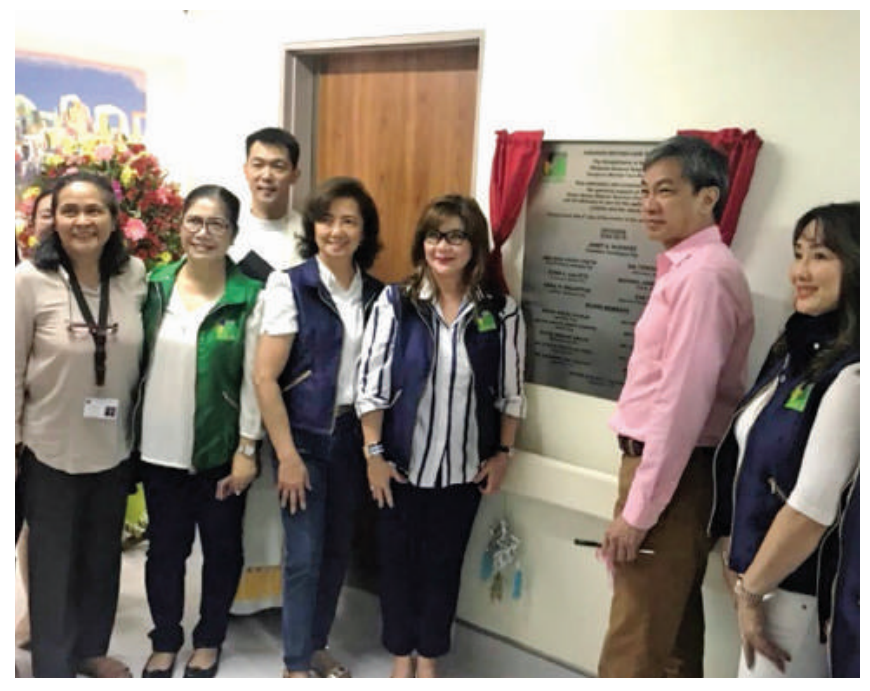

Figure 5. Inauguration of the Kangaroo Mother Care Room at the $4^{\text {th }}$ Floor of the PGH. At the foreground is Dr. Gerardo Legazpi and the officers of the Metro Manila Mayors' Spouses Foundation. Leftmost is Dr. Fay de Ocampo (Neonatologist, Division of Newborn Medicine, UP-PGH) and Dr. Lorna Abad (current Chair of Department of Pediatrics, UP-PGH).

the mentoring activity was assessed as a gratifying and calming activity for the students in the middle of their hectic schedules. Other mentoring groups subsequently requested a similar $\mathrm{KMC}$ encounter.

Lastly, PGH has forged linkages with international $\mathrm{KMC}$ groups by participating in international $\mathrm{KMC}$ workshops in Trieste, Italy, Rwanda, and Bogota, Colombia. Together with the College of Nursing and the KMC Foundation Philippines Inc., PGH co-hosted the First AsiaOceania KMC summit attended by more than $100 \mathrm{KMC}$ advocates from the Philippines, Indonesia, India, and Malaysia, Vietnam, and Mongolia in 2019 (Figure 8). KMC Foundation Philippines Inc. facilitated these opportunities.

Truly, KMC is a radical innovation in the field of medicine today. There are still bottlenecks in $\mathrm{KMC}$ implementation, but it has become a standard of care for stable low birth weight infants in the four years of PGH implementation through support from hospital leaders. Enrollment started at $57 \%$ before the start of the program in 2014 but has increased to $87 \%$ in $2017 . .^{9}$ With proper engagement and operation in the hospital; more lives will be saved. Optimal implementation of the program is thus imperative to obtain all the benefits of $\mathrm{KMC}$.

\section{CONCLUSION}

The KMC program is acceptable to all stakeholders. Compliance is also high, with a substantial increase in preterm and low birth weight infants enrolment after introducing the $\mathrm{KMC}$ program. However, adherence to the recommended $\mathrm{KMC}$ duration/day and data recording still need to be improved. Implementing strategies such as the presence of KMC champions, provision of meals and rooms to mothers, and the development of a computerbased data encoding program will all help improve KMC implementation.

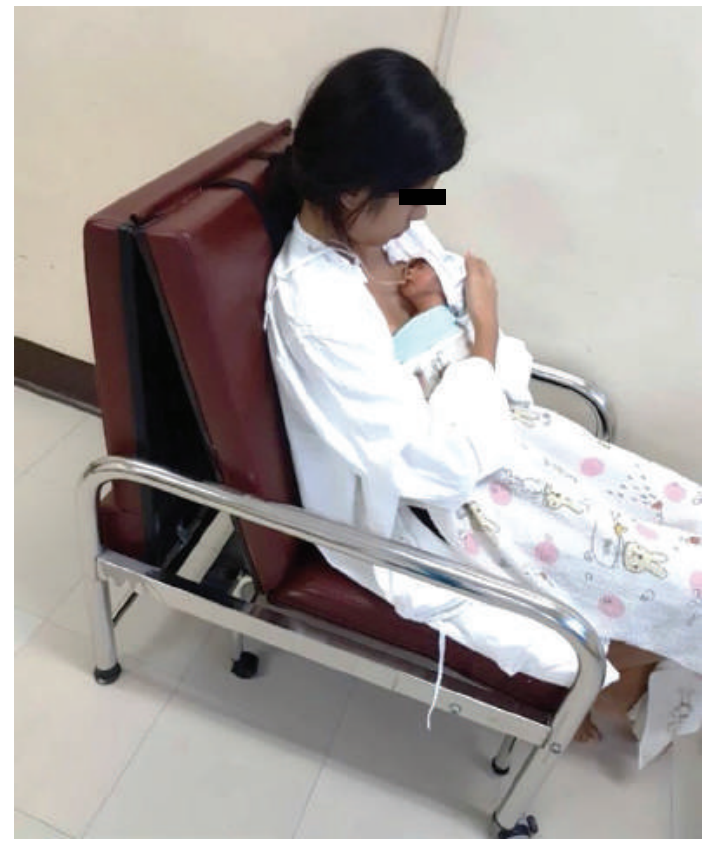

Figure 6. Adjustable KMC bed fabricated for PGH.

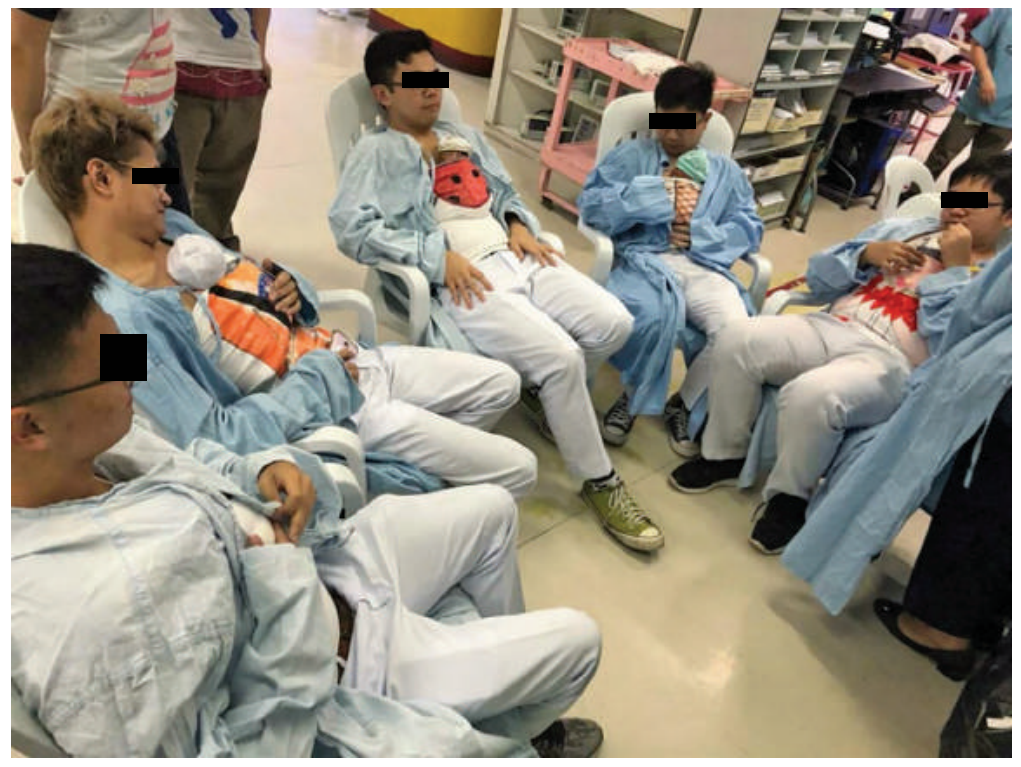

Figure 7. As part of a mentoring activity, medical students rendered KMC to the stable preterm infants with $\mathrm{KMC}$ tubes they designed themselves. 


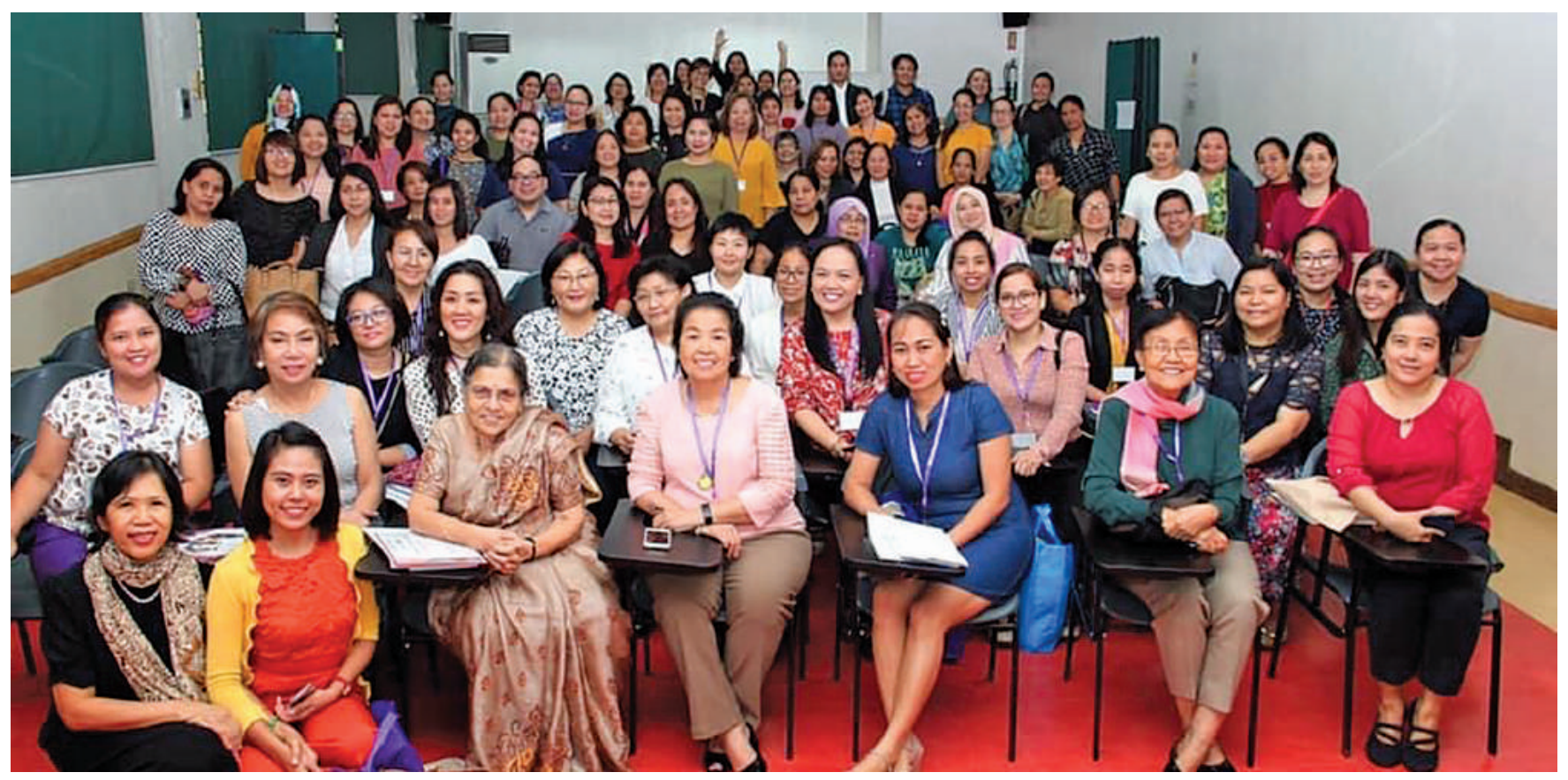

Figure 8. Facilitators and participants of the First AOK Summit with the Dr. Socorro Mendoza at the center (President of the KMC Foundation Philippines) and Dr. Shashi Vanni (Guest Speaker from India) on the left. Held at the Sotelo Hall, College of Nursing, University of the Philipinnes Manila.

\section{Statement of Authorship}

All authors participated in the data collection and analysis and approved the final version submitted.

\section{Author Disclosure}

All authors declared no conflicts of interest.

\section{Funding Source}

The study was funded by the Kangaroo Mother Care Foundation Philippines, Inc.

\section{REFERENCES}

1. March of Dimes, PMNCH, Save the Children, WHO. Born Too Soon: The Global Action Report on Preterm Birth. Eds. Howson CP, Kinney MV, Lawn JE. World Health Organization. Geneva, 2012 [Internet]. 2012 [cited 2018]. Available from: https://www.who.int/ pmnch/media/news/2012/201204_borntoosoon-report.pdf

2. Vesel L, Bergh AM, Kerber KJ, Valsangkar B, Mazia G, Moxon SG, et al. KMC Research Acceleration Group. Kangaroo Mother Care: a Multi-country Analysis of Health System Bottlenecks and Potential Solutions. BMC Pregnancy Childbirth. 2015;15 Suppl 2(Suppl 2):S5. doi: 10.1186/1471-2393-15-S2-S5. Epub 2015 Sep 11. PMID: 26391115; PMCID: PMC4577801.

3. Engmann C, Wall S, Darmstadt G, Valsangkar B, Claeson M; Participants of the Istanbul KMC Acceleration Meeting. Consensus on Kangaroo Mother Care Acceleration. Lancet. 2013 Nov 30;382(9907):e26-7. doi: 10.1016/S0140-6736(13)62293-X. Epub 2013 Nov 16. PMID: 24246562.

4. Annual Statistics, Section of Newborn Medicine, Department of Pediatrics, Philippine General Hospital 2014. Unpublished.

5. Annual Statistics, Section of Newborn Medicine, Department of Pediatrics, Philippine General Hospital 2015. Unpublished.
6. Annual Statistics, Section of Newborn Medicine, Department of Pediatrics, Philippine General Hospital 2016. Unpublished.

7. Castillo JE. DOH, PhilHealth to Launch Benefit Packages for Low-birthweight and Premature Babies in 2017. [Internet]. 2017 [cited 2018]. Available from: https://www.smartparenting.com.ph/ pregnancy/labor-and-childbirth/parents-can-avail-of-philhealths-new-premature-and-low-birth-weight-benefit-package-by2017-a1162-20161128

8. Bergh AM, de Graft-Johnson J, Khadka N, Om'Iniabohs A, Udani $\mathrm{R}$, Pratomo H, et al. The Three Waves in Implementation of Facilitybased Kangaroo Mother Care: a Multi-country Case Study from Asia. BMC Int Health Hum Rights. 2016 Jan 27;16:4. doi: 10.1186/ s12914-016-0080-4. PMID: 26818943; PMCID: PMC4730627.

9. Bilal SM, Tadele H, Abebo TA, Tadesse BT, Muleta M, W/Gebriel F, et al. Barriers for Kangaroo Mother Care (KMC) Acceptance and Practices in Southern Ethiopia: a Model for Scaling Up Uptake and Adherence Using Qualitative Study. BMC Pregnancy Childbirth. 2021 Jan 7;21(1):25. doi: 10.1186/s12884-020-03409-6. PMID: 33413193; PMCID: PMC7789316.

10. Cattaneo A, Amani A, Charpak N, De Leon-Mendoza S, Moxon S, Nimbalkar S, et al. Report on an International Workshop on Kangaroo Mother Care: Lessons Learned and a Vision for the Future. BMC Pregnancy Childbirth. 2018 May 16;18(1):170. doi: 10.1186/ s12884-018-1819-9. PMID: 29769056; PMCID: PMC5956892.

11. Chan G, Bergelson I, Smith ER, Skotnes T, Wall S. Barriers and Enablers of Kangaroo Mother Care Implementation from a Health Systems Perspective: a Systematic Review. Health Policy Plan. 2017 Dec 1;32(10):1466-75. doi: 10.1093/heapol/czx098. PMID: 28973515; PMCID: PMC5886293.

12. Smith ER, Bergelson I, Constantian S, Valsangkar B, Chan GJ. Barriers and Enablers of Health System Adoption of Kangaroo Mother Care: a Systematic Review of Caregiver Perspectives. BMC Pediatr. 2017 Jan 25;17(1):35. doi: 10.1186/s12887-016-0769-5. PMID: 28122592; PMCID: PMC5267363.

13. Annual Statistics, Section of Newborn Medicine, Department of Pediatrics, Philippine General Hospital 2017. Unpublished. 